\title{
Oncophagy: harnessing regulation of autophagy in cancer therapy
}

\author{
Justin S Gundara ${ }^{1,2, *}$, JingTing Zhao ${ }^{1}$, Bruce G Robinson ${ }^{1,3}$ and Stan B Sidhu ${ }^{1,2}$
}

${ }^{1}$ Cancer Genetics, Kolling Institute of Medical Research, ${ }^{2}$ Endocrine Surgical Unit and ${ }^{3}$ Department of Endocrinology, Royal North Shore Hospital, University of Sydney, Pacific Highway, St Leonards, Sydney, New South Wales 2065, Australia

(Correspondence should be addressed to S B Sidhu at Level 2, 69 Christie Street, St Leonards, Sydney, New South Wales 2065, Australia; Email: stansidhu@ nebsc.com.au)

*(J S Gundara is a Royal Australasian College of Surgeons, Roy McCaughey Surgical Research Fellow)

\begin{abstract}
Autophagy is an increasingly well-characterised process of cell component auto-digestion and recycling thought necessary for cellular subsistence. As we gain a more thorough understanding of the mechanisms underlying autophagy, its relevance to human disease and therapeutic potential are being clarified. This review summarises the evidence implicating autophagy in the pathogenesis and potential treatment of malignant disease. In addition, we explore the molecular role of microRNAs as key regulators in what we propose should now become known as 'oncophagy'.

Endocrine-Related Cancer (2012) 19 R281-R295
\end{abstract}

\section{Introduction}

Autophagy is a eukaryotic cell process of self-catabolic degradation originally described in the 1960's (Deter \& De Duve 1967). The term autophagy is derived from Greek terminology 'auto' (meaning self) and 'phagy' (eating) to describe the process of cell component autodigestion and recycling required for cellular survival. Beyond such origins, dual utility has also been proposed whereby autophagy plays somewhat of a Janus-like role in cell fate (Liu et al. 2011) being as important to cell death as it is to survival. Now that we are gaining a more thorough understanding of the mechanisms underlying autophagy, its relevance to human disease and burgeoning therapeutic potential are being unravelled (Ding et al. 2008, Chen et al. 2009, Li et al. 2009, 2012, Chen \& White 2011, Gundara et al. 2011). Here, we review the potential pathogenic, prognostic and therapeutic roles of autophagy within the context of human malignancy and with a special reference to microRNA-mediated regulation.

\section{Autophagy: the mechanism}

Cellular homoeostasis is a complex process dependent on close regulation of synthesis and degradation of both structural and functional elements. The ubiquitin-proteasome pathway is one such mechanism whereby cellular proteins can be broken down and disposed of or recycled. Alternatively, the autophagylysosome pathway maintains particular importance in management of longer lived or larger proteins, potentially including entire organelles such as mitochondria (Klionsky \& Emr 2000).

Autophagy has been sub-classified into three discrete sub-types (macro-, micro- and chaperonemediated autophagy), the central theme involving proteolysis of cytosol components at the lysosome (Glick et al. 2010). The majority of current scientific interest focuses on macroautophagy and its relation to cellular processes in health and disease (Glick et al. 2010). As such, the term autophagy is generally considered to be a reference to macroautophagy specifically (Klionsky et al. 2008).

Autophagy commences with the phagophore (an isolation membrane) that originates from the endoplasmic reticulum, golgi apparatus and endosomes. The phagophore consumes intra-cellular, cytoplasmic contents (damaged/dysfunctional DNA, cell organelles and proteins) and sequesters them within the doublemembrane autophagosome. This then fuses with the lysosome and lysosomal acid proteases induce degradation of autophagosomal contents (thence known as 
the autolysosome). The resulting component nucleotides, fatty acids and amino acids may then be recycled as cellular building blocks or removed as a waste product. Given such a mechanism, autophagy has been known as a 'recycling factory', enabling efficient energy production and removal of potentially toxic waste products of metabolic processes (Glick et al. 2010). Therein lies the importance of autophagy, which, when defective, can result in a multitude of human diseases (White et al. 2010).

\section{Autophagy: regulation}

Regulation of autophagy is complex. However, a basic understanding of the key regulatory steps is crucial to an understanding of potential autophagy-based biomarkers and therapies. In their review of autophagy regulation, Glick et al. (2010) describe five key points at which regulation is imposed:

1. phagophore formation,

2. Atg5-Atg12 conjugation, interaction with Atg16L and multimerisation at the phagophore,

3. LC3 processing and insertion into the extending phagophore membrane,

4. capture of random or selective targets for degradation,

5. fusion of the autophagosome with the lysosome, followed by proteolytic degradation by lysosomal proteases of engulfed molecules.

Numerous key regulators are involved in maintenance of the pathway from phagophore to autolysosome. Within this regulatory milieu reside $A T G$ s or autophagy-related genes and their associated Atgs (autophagy gene products). Our understanding of autophagy regulation was relatively superficial until the 1990's when $A T G$ s were discovered and have formed the crux of our understanding of regulatory processes since ( $\mathrm{Li}$ et al. 2012). Beclin-1 was the first ATG discovered to be of importance in mammalian autophagy (Liang et al. 1999) and there are now over 30 ATGs known to be involved. $A T G$ s (and their protein products) have subsequently been the target of specific investigation in an attempt to further delineate, and employ, the molecular utility of autophagy (Li et al. 2012).

Embedded within the myriad of regulatory processes governing autophagy is mammalian target of rapamycin (mTOR), which itself is part of the class I phosphatidylinositol 3-kinase (PI3K)/protein kinase B (PKB/Akt) signalling pathway. mTOR acts to inhibit autophagy under growth-promoting conditions, and repression of its activity has been found in states of nutrient deprivation and hypoxia, conditions also associated with autophagy (Yang \& Klionsky 2010). More specifically, class I PI3K is activated by growth factor receptors, which then lead to downstream mTOR activation and ultimately autophagy inhibition (Yang \& Klionsky 2010). Thus, it can be reasoned that in states of nutrient deprivation, a lack of growth factors will release mTOR inhibitory signalling and result in autophagy induction to recycle cellular constituents and maintain cell viability.

An understanding of the mechanistic pathways involved is of great interest as it opens opportunities for potential therapeutic intervention. The ability to manipulate these complex pathways is, however, dependent on an understanding of the clinical importance of autophagy in disease, and cancer in particular. Beyond a homoeostatic role, autophagy has been shown to be of importance in normal development (Kuma et al. 2004), and from a clinicopathologic viewpoint, it has been associated with neurodegenerative (Yang \& Mao 2010), metabolic (Jung \& Lee 2010), infectious (Gutierrez et al. 2007), inflammatory (White et al. 2010) and malignant diseases (Chen et al. 2009, White et al. 2010, Li et al. 2012).

The harnessing of a self-catabolic process like autophagy against malignancy is a novel concept and is the one that is fast gaining broad scientific and clinical interest (White \& DiPaola 2009, Ravikumar et al. 2010, White et al. 2010, Li et al. 2012). The remainder of this review focuses primarily on our current understanding of the role of autophagy in cancer biology and the steps that have already been taken to utilise this process as a potential therapeutic strategy in cancer treatment.

\section{Autophagy: role in oncogenesis and cancer therapy}

Currently, conflicting evidence regarding the role of autophagy in tumour biology has led to a likening of this process to a double-edged sword (White \& DiPaola 2009). Autophagy has been shown to be both inhibitory and beneficial to the malignant process (Ravikumar et al. 2010). Some investigators have suggested that it may suppress early tumour development, serving as an anti-neoplastic, biological defence mechanism in the early stages of oncogenesis (Karantza-Wadsworth et al. 2007), while paradoxically supporting tumour cell survival later, once true malignancy has evolved (White et al. 2010, Yang \& Kimmelman 2011). Enhanced sensitivity of cancer 
cells to chemo- and radiotherapy has also been associated with enhanced autophagic flux (Mukubou et al. 2010), although discerning objectively what reflects an efficacious therapeutic effect, rather than evidence of collateral cellular stress is a contentious issue (Klionsky et al. 2008, Shen et al. 2012). Additionally, direct quantification of basal tumour tissue autophagic flux has also proven difficult and has led some investigators to suggest that direct evidence linking reduced autophagic flux to tumorigenesis is still lacking (Chen \& Debnath 2010). These observations reinforce the notion that the role of autophagy in oncogenesis is not only highly context dependent (White et al. 2010), but it is also still poorly understood.

\section{Autophagic cell death}

The association between oncogenesis and autophagy is typified by the finding of several ATGs and associated proteins that have been touted as potential tumour suppressors. Beclin-1 (Qu et al. 2003), PTEN (Arico et al. 2001) and 553 (Feng et al. 2005) have been found to be significant in autophagy pathways, with Beclin-1 gene suppression, for example, being linked directly to tumorigenesis (Qu et al. 2003). More specifically, reduced Beclin-1 expression has been identified in sporadic human breast cancers (Liang et al. 1999), higher grade brain tumours (Miracco et al. 2007) and epithelial ovarian cancers (Shen et al. 2008). The Beclin-1 genomic locus (chromosome 17q21) has also been shown to be deleted in up to $50 \%$ of breast (Saito et al. 1993), 75\% of ovarian (Russell et al. 1990, Saito et al. 1993) and 40\% of prostate (Gao et al. 1995) cancers. Conversely, elevated levels of Beclin-1 expression correlate significantly with disease-free and overall survival in colon (Li et al. 2009), hepatocellular (Ding et al. 2008) and oesophageal (squamous cell) cancers (Chen et al. 2009), and functional experimentation demonstrates that Beclin-1 overexpression can reduce in vitro colon (Koneri et al. 2007) and cervical (Wang et al. 2007) cancer cell proliferation and in vivo xenograft tumour growth (Wang et al. 2007). This effect is reversed when Beclin-1 is suppressed using RNA interference methods (Wang et al. 2007).

It has also been suggested that the tumour suppressor role of PTEN is enacted indirectly through positive regulation of autophagy (Arico et al. 2001). This is thought to occur secondary to PTEN inhibition of the PI3K/Akt pathway, as originally demonstrated in a colon cancer cell line by Arico et al. (2001). Consistent with this mechanism, heterozygous loss of PTEN in mice has been shown to lead to breast and thyroid tumours, among others (Kishimoto et al. 2003). Additionally, PTEN mutations have also been identified in a range of hereditary cancer syndromes (Pezzolesi et al.2008) and aggressive sporadic malignancies, such as castration-resistant prostate cancer (Grasso et al. 2012). A similar positive regulatory role in autophagy has also been proven for the commonly implicated tumour suppressor, p53 (Feng et al. 2005).

These findings support the theory that ongoing and continual autophagic flux may be tumoricidal and necessary to avoid neoplastic transformation, during the earliest stages of cancer initiation (Shen et al. 2008, White et al. 2010). However, if autophagy fails to maintain this housekeeper function, neoplasia ensues. This effect has been demonstrated in breast cancer and defines the role of Beclin-1 as a haplo-insufficient tumour suppressor (Karantza-Wadsworth et al. 2007). Having demonstrated such an effect, Karantza-Wadsworth et al. (2007) concluded that defective autophagy leaves cells susceptible to metabolic stress and DNA damage that ultimately leads to genomic instability and cancer initiation. It may be that many tumour suppressors, not unlike Beclin-1, PTEN or p53, exert their 'suppressor' effect through such an autophagic avenue.

In keeping with this theoretical continuum, associations between therapeutic response to cytotoxic agents and elevations in markers of autophagic flux have led some investigators to claim that iatrogenic autophagy induction may be a valid therapeutic strategy, by taking advantage of what was previously labelled 'autophagic cell death' (Ullen et al. 2010, Abraham et al. 2011). Ullen et al. (2010), in examining the in vitro efficacy of the tyrosine kinase inhibitor, sorafenib on prostate cancer cells, identified a dosedependent impact on cell viability in parallel with evidence of increased autophagic flux. This led them to suggest that autophagy manipulation (in combination with established therapies) may be a valid approach to treating cancer.

Numerous other malignancies have also been investigated regarding the potential therapeutic harnessing of autophagic cell death. Using a variety of agents, claims of autophagic cell death have been made in a vast range of malignancies including hepatocellular carcinoma (HCC; Wang et al. 2010), oestrogen receptor-negative breast cancer (Vanderlaag et al. 2010), non-small-cell lung cancer ( $\mathrm{Li}$ et al. 2010b), ovarian cancer (Le et al. 2010), pancreatic cancer (Mujumdar et al. 2010), gastric cancer (Hashimoto et al. 2008), malignant glioma (Takeuchi et al. 2005) and papillary thyroid cancer (Lin et al. 2009). Many of these investigations demonstrate 
an apoptosis-independent therapeutic in vitro effect on cellular proliferation in association with elevations in markers of autophagy. In reasoning such results, investigators have previously concluded that autophagic cell death is the mechanism by which therapeutic efficacy is being enacted.

The role of the tyrosine kinase receptor pathway and autophagy has also been highlighted more recently by Xu \& Weihua (2011), who demonstrated that EGFR receptor knockdown leads to autophagy induction. However, and in direct contrast to Ullen et al., they concluded that the beneficial effect on prostate cancer cells related to EGFR protein suppression rather than inhibition of tyrosine kinase activity.

Table 1 summarises a broad, representative selection of basic scientific and clinicopathologic literature examining the pathophysiological, prognostic and therapeutic themes of what can now be labelled 'oncophagy' (i.e. cancer-related autophagy).

\section{The alternative hypothesis}

One of the lasting problems in identification of what may be considered autophagic cell death is confirming that this is the definitive, lethal process cells are committing to, rather than being an observation of a collateral response to stress that accompanies cellular demise. This is a present-day challenge for autophagy investigators and is a concept that has recently been challenged strongly (Shen et al. 2012).

Shen et al. (2012) questioned whether or not autophagic cell death exits at all. Following exhaustive experimentation involving treatment of osteosarcoma cells with 1400 different agents, they were unable to identify definitive evidence of autophagic cell death (Shen et al. 2011). They concluded that markers of autophagic flux may well be present within the context of experimentation attempting to demonstrate a therapeutic effect, but this does not constitute evidence of cell death 'by' autophagy, but rather, death 'with' autophagy. This is an important point, and one that has led to abandonment of older terminology whereby autophagy was otherwise known as programmed cell death type II (Kroemer et al. 2009). Despite this, the possibility of autophagy still being intimately involved in 'lethal signalling' pathways towards cell death is likely, but the precise role it plays requires further investigation and definition.

Given that the case for induced autophagic cell death is becoming less compelling, an alternative hypothesis requires consideration. In keeping with the homoeostatic role of autophagy in cellular health, there is evolving evidence that this may also be a potent strategy employed by tumour cells to ensure survival, thus paving the way for therapeutic manipulation (White \& DiPaola 2009). White \& DiPoala articulate this concept well by suggesting that tumour cells, with their rapid proliferation and poor blood supply, are some of the most metabolically stressed. This is justified by data showing that tumour tissues generally possess an increased state of autophagic flux (Degenhardt et al. 2006), therefore supporting the theory of a heightened reliance on mechanisms such as autophagy for survival and ultimately enhanced levels of stress tolerance when compared to cells in health (White \& DiPaola 2009). Suppressing or removing this tumour cell survival mechanism would thus seem an attractive therapeutic manoeuvre.

The at times conflicting, two faced nature of autophagy involvement in oncogenesis is exemplified by the tumour suppressor gene ARHI (DIRAS3) (Lu et al. 2008). In attempting to explore ARHI gene suppression in ovarian cancer, Lu et al. induced re-expression of $A R H I$ in a cell culture model and demonstrated cell death in association with autophagy induction. When cells were xenotransplanted into a mouse model without $A R H I$ induction, tumours predictably grew, and when $A R H I$ was induced tumour growth was inhibited, as expected. When ARHI induction was withdrawn after 30 days, tumours began to grow promptly indicating that xenografted cells had remained dormant but still viable and capable of proliferation. Additional experimentation with autophagy blockade (using choloroquine) led to retarded tumour growth following $A R H I$ induction withdrawal. Cells were again viable but apparently dormant. The authors suggest that this is indicative of the establishment of an adaptive dormancy state that is dependent on ARHI-mediated autophagy. Lu et al. concluded that autophagy, while maintaining an efficacious tumoricidal effect in vitro, seemingly appears to aid maintenance of tumour cell dormancy in the in vivo model, thus implicating it as a tumour cell survival strategy. This provided some of the first evidence of therapeutic benefit when employing autophagy blockade, rather than induction, in a model of established malignancy.

A role for autophagic tumour cell survival has also been investigated in HCC cell lines and xenografts (Ding et al. 2011). Ding et al. treated HCC cell lines with oxaliplatin, a known chemotherapeutic agent efficacious in HCC. Treated cells and mouse model xenografts demonstrated evidence of autophagy induction in association with cell death and tumour 
regression. However, when treatment was supplemented with autophagy blockade (using pharmacological and small interfering RNA methods), oxaliplatin treatment was potentiated with an increase in cell death and tumour regression being observed. These results suggest that autophagy may well be an adaptive tumour cell survival mechanism rather than promoting cell death. Similar findings have been reported by $\mathrm{Li}$ et al. (2010a) when examining the increased effect of 5-FU on colon cancer cell lines and xenografts with autophagy blockade (employing 3-methyladenine and siRNA to Atg7). Interestingly, this was also observed in association with an increase in flux through the apoptotic pathway, implying a degree of crosstalk between these two pathways of cell fate, a link that has been suggested previously (Shen et al. 2011). In exploring the cross talk between apoptosis, autophagy and necrosis, Shen et al. (2012) also showed enhanced cellular toxicity during treatment with autophagy blockade (using siRNA to ATG5 and $A T G 7$ ) to further reinforce the theory of autophagy being a cytoprotective mechanism. The alternative hypothesis therefore follows: namely that if this cytoprotective mechanism is disabled, tumour cells are vulnerable to toxic insults and death.

This novel theory has been expanded on both in the laboratory and also clinically through the commencement of several clinical trials that are attempting to exploit pharmacologic autophagy blockade in combination with recognised chemotherapeutics for cancer. Adjuvant autophagy blockade has been shown to be therapeutic during in vitro studies investigating various treatments for myeloid leukaemia (Bellodi et al. 2009), colon cancer (Carew et al. 2010), glioma (Lomonaco et al. 2009) and breast cancer (Vazquez-Martin et al. 2009). In addition, and while it is beyond the scope of this review to go into detail, it is important to note that such discoveries are now being translated into several phase II trials that are underway and are employing choloroquine as the autophagy blocking agent of choice. Efficacy in a number of cancer types is being explored, ranging from haematological malignancies to solid adult tumours, prostate and lung cancer (White \& DiPaola 2009). The specifics of these trials are summarised in White \& DiPaola (2009).

Thus, it would appear that there are a number of important factors within the realm of experimental therapeutic oncophagy that investigators should be aware of. The cell, tissue or tumour type; stage of malignant transformation and nature of treatment employed are important contextual factors of significance. The White et al. group focus on these concepts in their eloquent reviews of autophagy in inflammation and cancer (White \& DiPaola 2009, White et al. 2010). They also promote the 'double-edged' nature of autophagy by suggesting that autophagy generally promotes cellular survival and an anti-neoplastic housekeeping function that, when defective, is potentially carcinogenic. More specifically, they cite the inability of an impaired autophagic process to clear damaged proteins and organelles, leaving a toxic stimulus within the cellular milieu that may induce genetic instability and ultimately malignant transformation. They go on to suggest that while autophagy blockade may be therapeutically useful in managing progression of malignant disease, it may also be logical and advantageous to induce autophagy as a protective strategy in the early stages of pre-malignant transformation. This could even be considered in a prophylactic context.

The supposed paradoxical nature of autophagy manipulation may therefore be a redundant concept, or rather, it should be recognised that autophagy is a dynamic biological process. Autophagy induction is prophylactic before malignancy evolves, and blockade is therapeutic for established disease, which may well be the mantra of oncophagy as we continue exploration in an attempt to harness this normal physiological process for management of a highly abnormal one.

\section{Novel therapeutic approaches: the autophagamiR}

While the genetic and molecular basis of autophagy is now coming under increasing scrutiny, there remains a dearth of knowledge regarding the influence of microRNA's (miRNAs). miRNAs are short ( 22nt) non-coding RNAs that affect regulation at a posttranscriptional level through target mRNA suppression (Shenouda \& Alahari 2009). This is achieved through recognition of the miRNA response element present in the $3^{\prime}$ UTR domain of the target mRNA. Given the exponential increase in interest regarding miRNA profiles of malignant diseases (and associated mRNA targets), the question regarding miRNA regulation of autophagy within the cancer context requires clarification (Gundara et al. 2011). More specifically, the negative regulatory nature of miRNA function is especially attractive within the domain of oncophagy, given increasing interest in strategies employing blockade of cytoprotective, autophagic flux.

One of the earliest studies considering this issue demonstrated a role for miR-30a targeting of Beclin-1 and subsequently autophagic flux in breast, brain and 


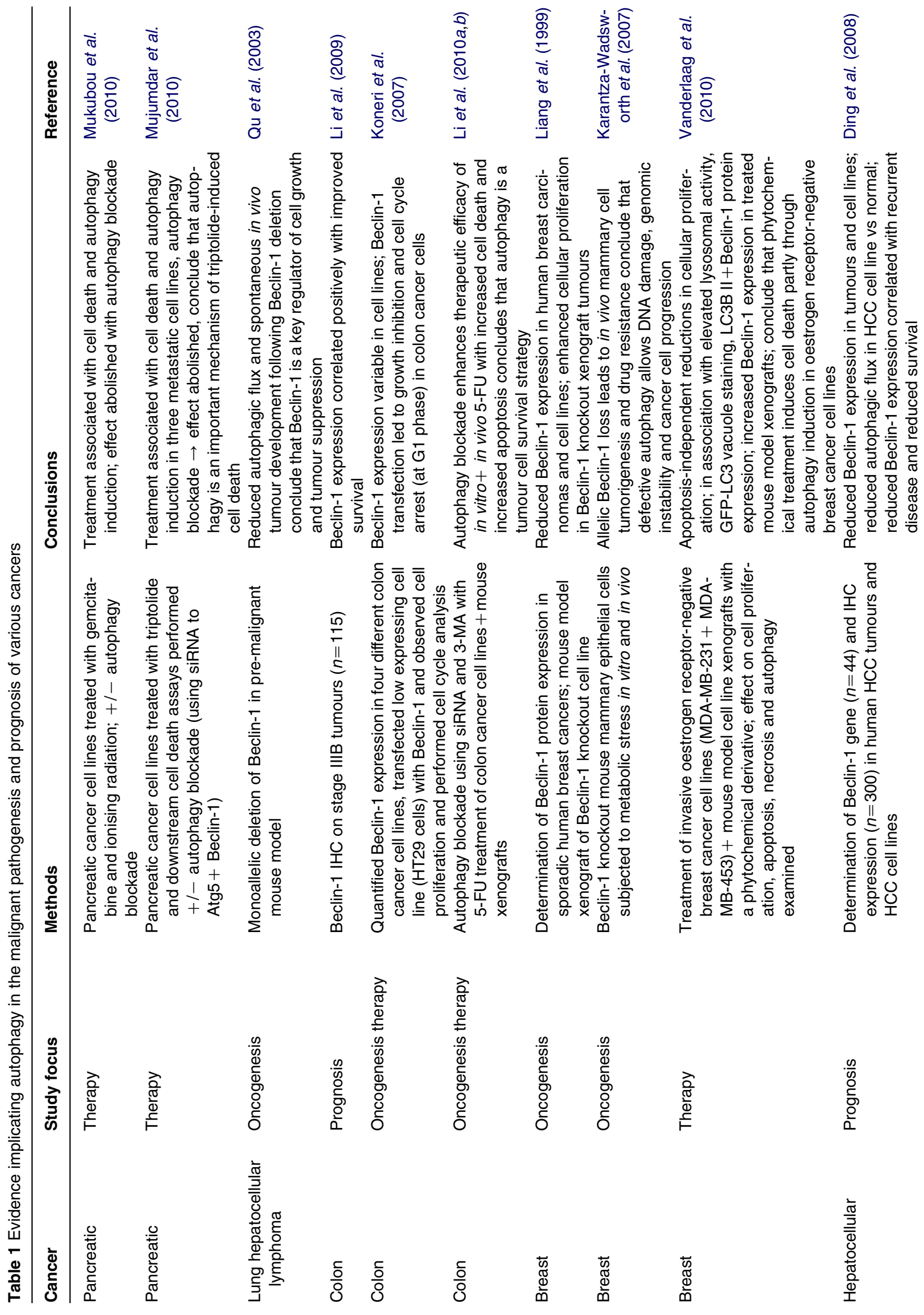




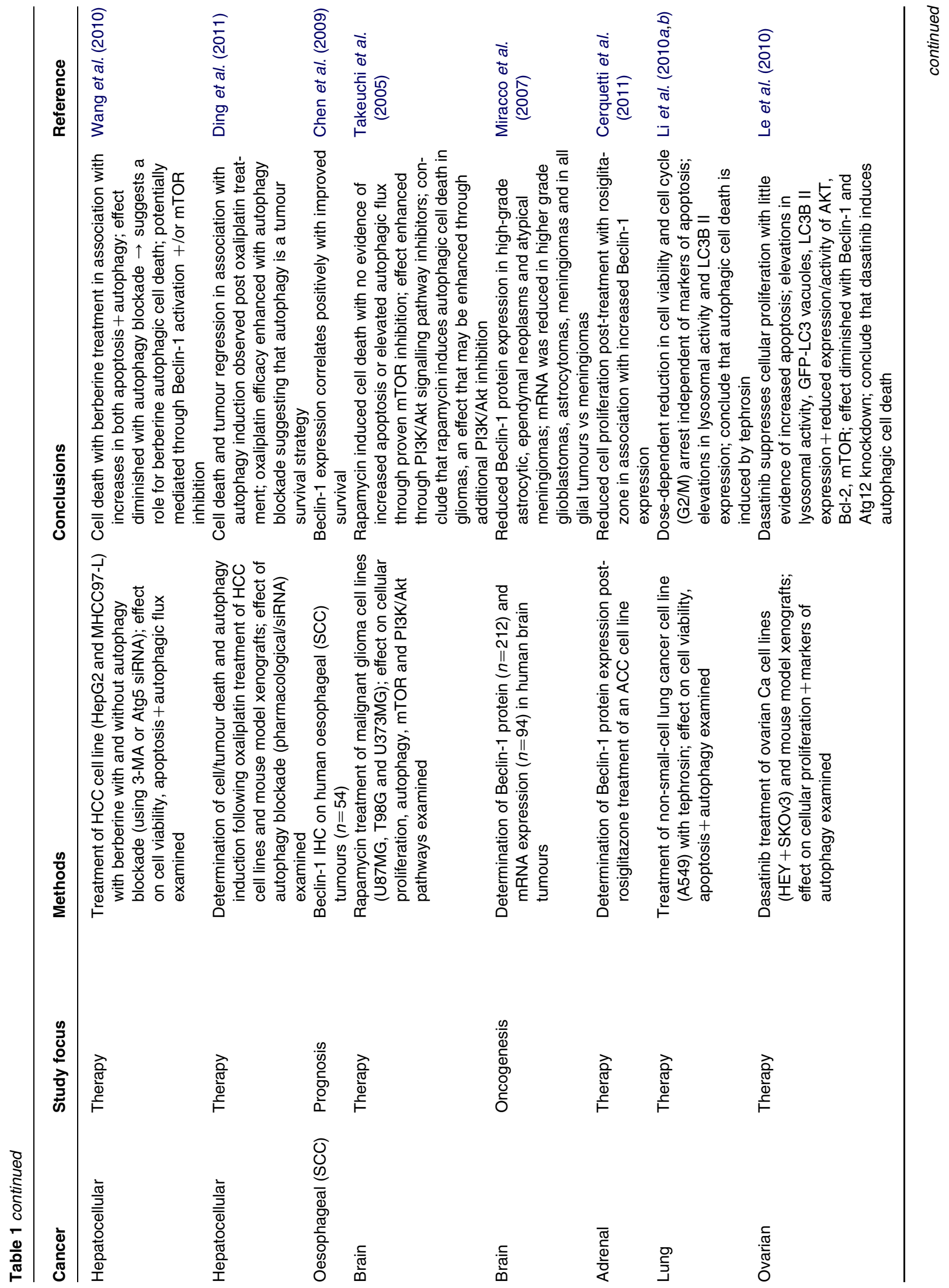




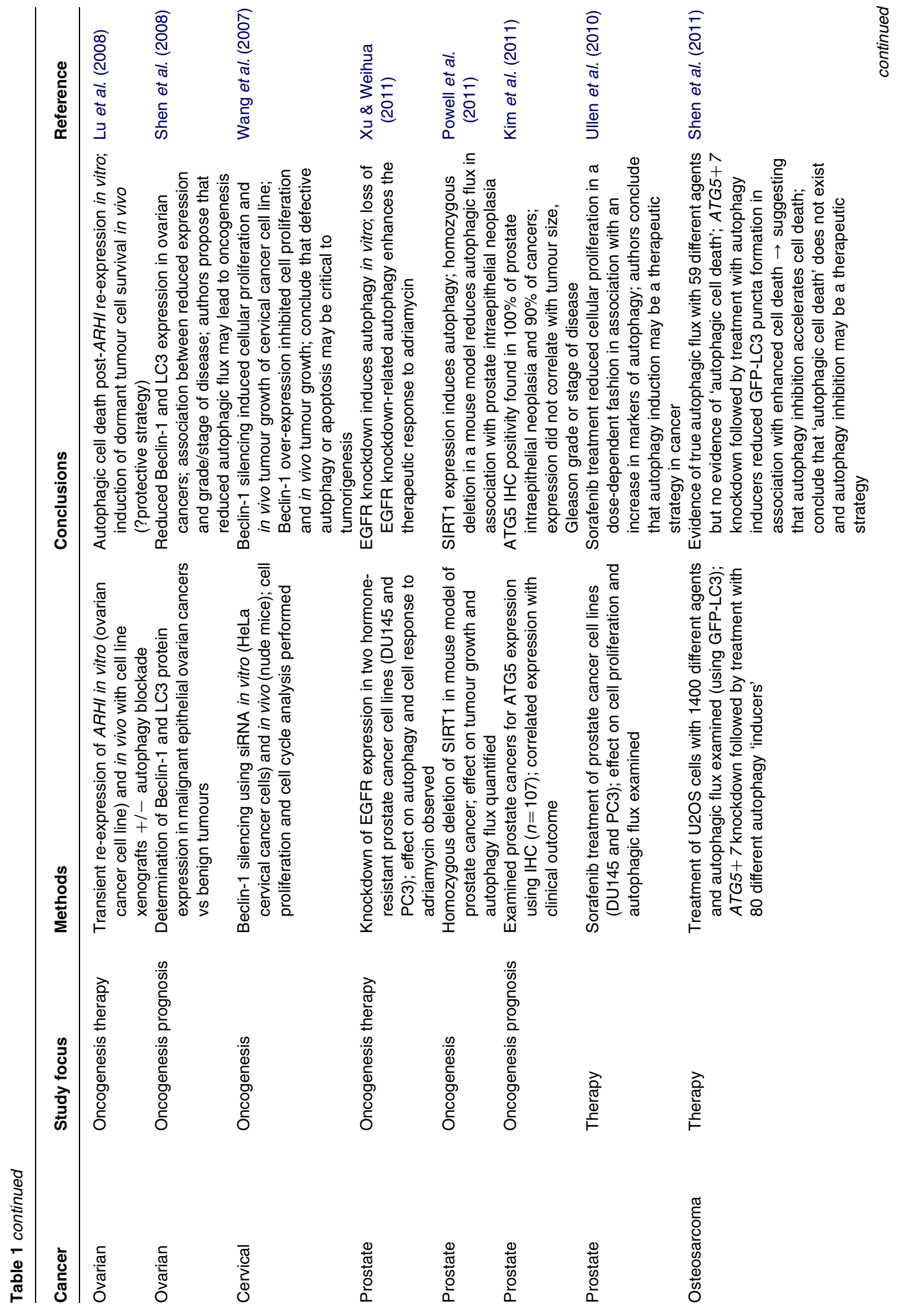




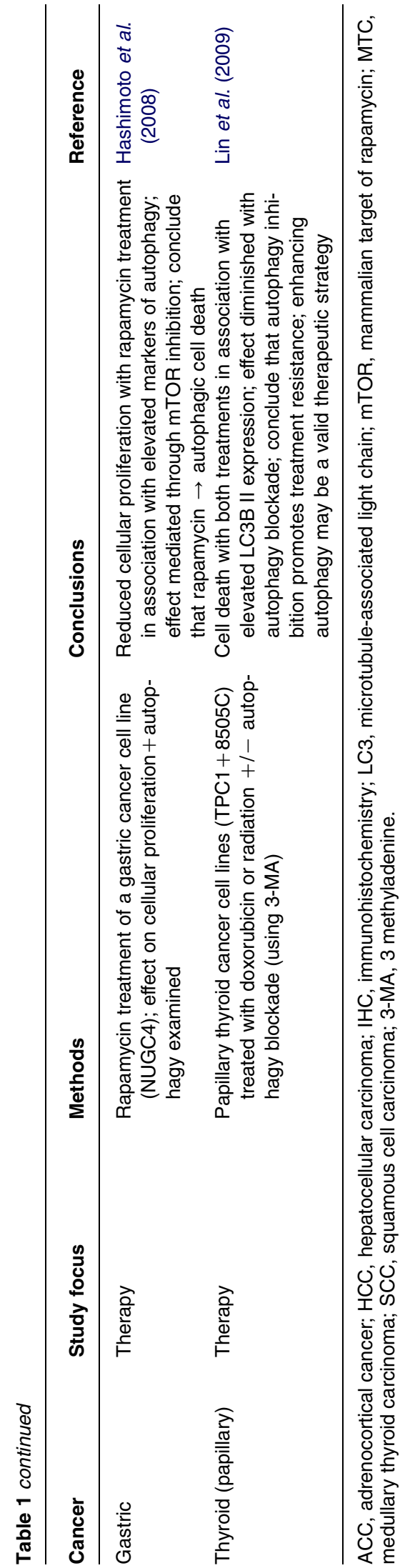

lung cancer cell lines (Zhu et al. 2009). Functional experimentation showed that miR-30a negatively regulated Beclin-1 mRNA and protein expression and subsequently inhibited autophagic flux. This effect was maintained despite attempts to induce autophagy with rapamycin. Unfortunately, the downstream influence on cellular proliferation was not examined in this study, making it difficult to comment further regarding the theory of autophagy blockade as a therapeutic strategy. However, Zou et al. (2012) have recently confirmed this hypothesis by showing that miR-30a inhibition of Beclin-1 can enhance the efficacy of platinum-based chemotherapy in vitro, effectively sensitising cancer cells to therapy through active inhibition of autophagy.

Subsequent to the original studies of Zhu et al., Frankel et al. (2011) have demonstrated that miR-101 negatively regulates autophagy in MCF-7, breast cancer cells. Overexpression of miR-101 was shown to inhibit autophagy, and when knocked down, autophagy induction was observed. Importantly, Frankel et al. were also able to identify several miR-101 targets. This was achieved through an mRNA array study, $48 \mathrm{~h}$ post-miR-101 transfection of MCF-7 cells. Following filtering, normalisation, statistical analyses and qPCR validation, 14 potential targets were identified. Those deemed to be of interest within the context of miRNA regulation of autophagy (STMN1, RAB5A and ATG4D) were then knocked down using siRNAs in an attempt to mimic the effect of miR-101 on MCF-7 cells. As hypothesised, knockdown of gene targets inhibited basal levels of autophagy and also blocked rapamycin-induced autophagy to a similar extent to that induced by miR-101 transfection. These studies reinforce the therapeutic benefit of miRNA-mediated inhibition of autophagy.

miR-375 has also been shown to regulate the response to hypoxic stress-induced autophagy (Chang et al. 2012). Using HCC cell lines and mouse model xenografts, Chang et al. demonstrated that hypoxia induced evidence of autophagic flux that was abolished with transfection of a miRNA shown to be downregulated in HCC cell lines (i.e. miR-375). The ability of cancer cells to employ autophagy as a tumour cell survival strategy was similarly compromised following miR-375 transfection and numbers of viable cells were reduced accordingly. Additionally, a predicted miR-375 target, $A T G 7$, was validated through luciferase reporter experimentation.

Similar findings that support the importance of what are now known as autophagamiRs (Gundara et al. 2011) have been reported more recently, further 


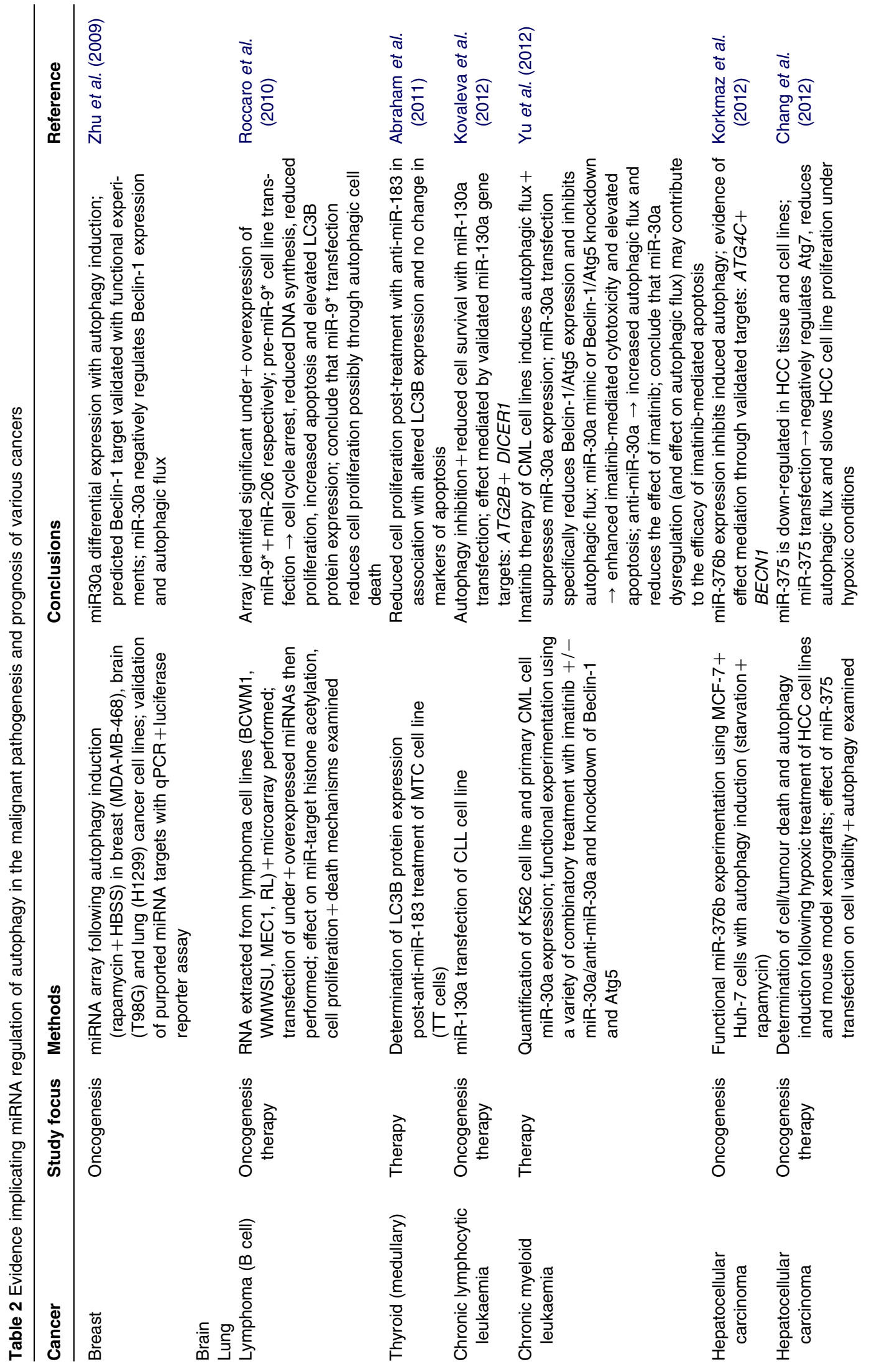




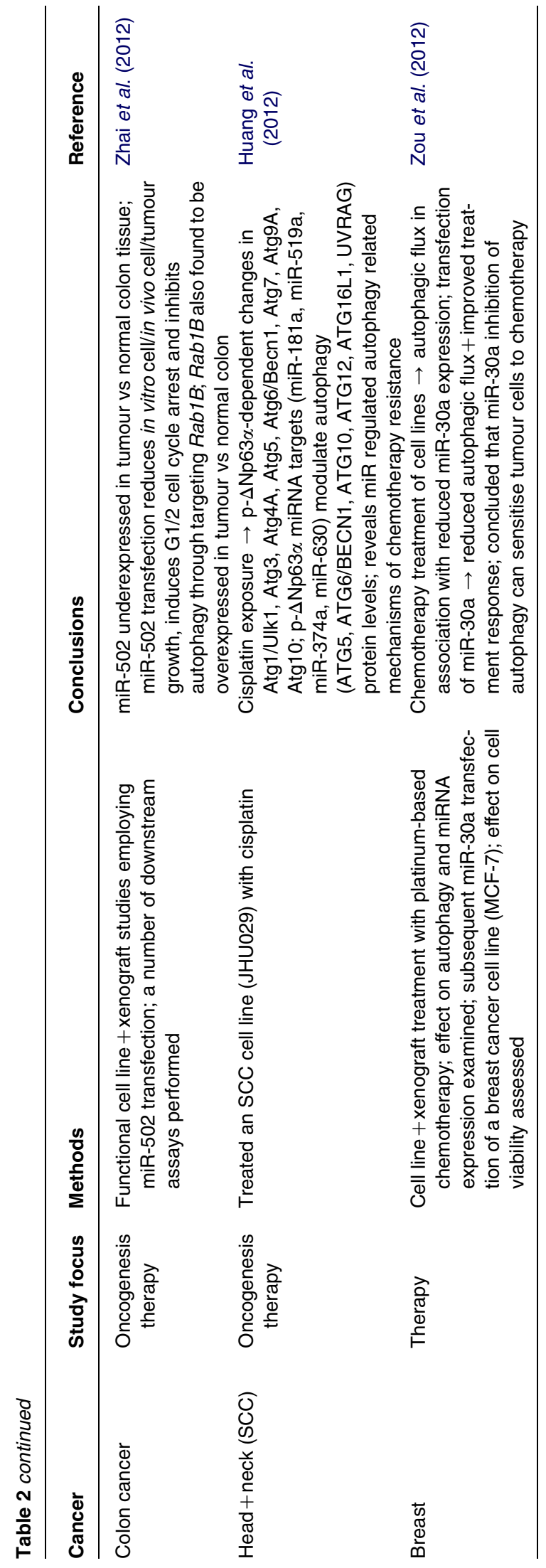

validating this concept. Roles for miR-130a and miR-143 have been demonstrated in chronic lymphocytic leukaemia, with autophagy inhibition shown to be affected through repression of validated miR-130a gene targets, $A T G 2 B$ and DICERI (Kovaleva et al. 2012). miR-376b has also been shown to inhibit autophagy through $A T G 4 C$ and BECN1 repression in both breast cancer (MCF-7) and hepatoma (Huh-7) cell lines (Korkmaz et al. 2012). Comparable results have been reproduced by other groups using miR-181a, a result facilitated through ATG5 inhibition of a cisplatin-resistant squamous cell carcinoma cell line (Huang et al. 2012). Furthermore, colon cancer miR-502 under-expression, when 'replaced' using both in vitro and in vivo models, has shown evidence of autophagy inhibition and cell death through RablB repression (shown to be overexpressed in human colon cancer tissue samples) (Zhai et al. 2012). Lastly, a role for miR-9* regulation of autophagy has also been reported as one mechanism for cell toxicity in a cell culture model of Waldentsrom macroglobulinaemia; a form of B-cell lymphoma (Roccaro et al. 2010).

Huang et al., while demonstrating the link between miR-181a and ATG5, were also able to identify the role of autophagamiR in drug resistance. More specifically, they showed that exposure of a squamous cell carcinoma cells to cisplatin resulted in altered expression of a number of markers of autophagic flux, an effect mediated by $\mathrm{p}-\Delta \mathrm{Np} 63 \alpha$-dependent transcriptional regulation. Additionally, miR-181a was also shown to be a downstream p- $\Delta$ Np63 $\alpha$ effector target. Taken together, these results have revealed some of the first evidence of autophagamiR-mediated drug resistance mechanisms, thus enabling further work geared towards manipulation of these evolving treatment resistance strategies.

Similarly, the studies of Zou et al. have also showed that platinum-based chemotherapy leads not only to evidence of autophagic flux but also to reduced miR-30a tumour expression. Forced expression of miR-30a in cell culture and xenograft-treated cells subsequently resulted in enhanced treatment response, in association with reduced autophagic flux (Zou et al. 2012). Similar themes have been explored by Yu et al. (2012) in chronic myeloid leukaemia, whereby a role for miR-30a-induced autophagic repression in enhancing the response to imatinib therapy (an autophagy inducing insult) has also been proven. These findings reaffirm suggestions of tumour cell drug resistance mechanisms being reliant on autophagy, which, when manipulated through an improved understanding of miRNA regulation, results in enhanced treatment sensitivity. 
Clearly then, investigation of miRNAs that govern autophagy is well and truly in evolution and the present body of evidence is in favour of miRNA inhibition of autophagic flux as the mechanism of therapeutic benefit. Not only is autophagy an important process within tumour biology but miRNAs are now becoming important players in regulation of what may ultimately be shown to be a fundamental tumour cell survival strategy and one that may even be of importance in acquired drug resistance. The translational impact of these findings is now more obvious than ever and miRNAs may represent the key to clinical application of the principles governing oncophagy. A summary of studies focusing on investigation of the autophagamiR in cancer is detailed in Table 2.

\section{Conclusion}

Our philosophical interpretation of the aforementioned findings is of great significance. Focussing too heavily on the inner conflict regarding whether autophagy is pro- or anti-tumour is to lose sight of the ultimate objective, namely, harnessing therapeutic potential regardless of the specific mechanism or method employed. An alternative viewpoint would maintain that these results do not provide contradictory evidence, but rather unveil the theme of manipulating autophagy dynamics within context, meaning that the self-catabolic process should not necessarily be inextricably linked to cell death, but rather be seen as a process that is intrinsic to the cell's response to any foreign (or native) insult. Autophagy induction has been associated with therapeutic benefit and this may well prove to be an effective strategy, but we must also take advantage of a context in which autophagy is cytoprotective and employ blockade as an alternative treatment strategy. The negative regulatory nature of miRNAs makes them a logical and important candidate for blockade initiation. The overriding challenge, however, lies in identifying which approach to employ, and when.

\section{Declaration of interest}

The authors declare that there is no conflict of interest that could be perceived as prejudicing the impartiality of the review.

\section{Funding}

Research Support: this work was supported by the by the Royal Australasian College of Surgeons (Sir Roy McCaughey Surgical Research Fellowship) and the Cancer Institute of NSW (Northern Translational Cancer Research Unit Scholarship).

\section{References}

Abraham D, Jackson N, Gundara JS, Zhao J, Gill AJ, Delbridge L, Robinson BG \& Sidhu SB 2011 MicroRNA profiling of sporadic and hereditary medullary thyroid cancer identifies predictors of nodal metastasis, prognosis, and potential therapeutic targets. Clinical Cancer Research 17 4772-4781. (doi:10.1158/1078-0432.CCR11-0242)

Arico S, Petiot A, Bauvy C, Dubbelhuis PF, Meijer AJ, Codogno P \& Ogier-Denis E 2001 The tumor suppressor PTEN positively regulates macroautophagy by inhibiting the phosphatidylinositol 3-kinase/protein kinase B pathway. Journal of Biological Chemistry 276 35243-35246. (doi:10.1074/jbc.C100319200)

Bellodi C, Lidonnici MR, Hamilton A, Helgason GV, Soliera AR, Ronchetti M, Galavotti S, Young KW, Selmi T, Yacobi R et al. 2009 Targeting autophagy potentiates tyrosine kinase inhibitor-induced cell death in Philadelphia chromosome-positive cells, including primary CML stem cells. Journal of Clinical Investigation 119 1109-1123. (doi:10.1172/JCI35660)

Carew JS, Medina EC, Esquivel JA II, Mahalingam D, Swords R, Kelly K, Zhang H, Huang P, Mita AC, Mita MM et al. 2010 Autophagy inhibition enhances vorinostat-induced apoptosis via ubiquitinated protein accumulation. Journal of Cellular and Molecular Medicine 14 2448-2459. (doi:10.1111/ j.1582-4934.2009.00832.x)

Cerquetti L, Sampaoli C, Amendola D, Bucci B, Masuelli L, Marchese R, Misiti S, De Venanzi A, Poggi M, Toscano V \& Stigliano A 2011 Rosiglitazone induces autophagy in H295R and cell cycle deregulation in SW13 adrenocortical cancer cells. Experimental Cell Research $\mathbf{3 1 7}$ 1397-1410.

Chang Y, Yan W, He X, Zhang L, Li C, Huang H, Nace G, Geller DA, Lin J \& Tsung A 2012 miR-375 inhibits autophagy and reduces viability of hepatocellular carcinoma cells under hypoxic conditions. Gastroenterology 143 177.e8-187.e8. (doi:10.1053/j.gastro.2012.04.009)

Chen N \& Debnath J 2010 Autophagy and tumorigenesis. FEBS Letters 584 1427-1435. (doi:10.1016/j.febslet. 2009.12.034)

Chen H-Y \& White E 2011 Role of autophagy in cancer prevention. Cancer Prevention Research 4 973-983. (doi:10.1158/1940-6207.CAPR-10-0387)

Chen Y, Lu Y, Lu C \& Zhang L 2009 Beclin-1 expression is a predictor of clinical outcome in patients with esophageal squamous cell carcinoma and correlated to hypoxiainducible factor (HIF)- $1 \alpha$ expression. Pathology Oncology Research 15 487-493. (doi:10.1007/s12253-0089143-8)

Degenhardt K, Mathew R, Beaudoin B, Bray K, Anderson D, Chen G, Mukherjee C, Shi Y, Gelinas C, Fan Y et al. 2006 Autophagy promotes tumor cell survival and restricts necrosis, inflammation, and tumorigenesis. Cancer Cell 10 51-64. (doi:10.1016/j.ccr.2006.06.001) 
Deter RL \& De Duve C 1967 Influence of glucagon, an inducer of cellular autophagy, on some physical properties of rat liver lysosomes. Journal of Cell Biology 33 437-449. (doi:10.1083/jcb.33.2.437)

Ding Z-B, Shi Y-H, Zhou J, Qiu S-J, Xu Y, Dai Z, Shi G-M, Wang X-Y, Ke A-W, Wu B et al. 2008 Association of autophagy defect with a malignant phenotype and poor prognosis of hepatocellular carcinoma. Cancer Research 68 9167-9175. (doi:10.1158/0008-5472.CAN-08-1573)

Ding ZB, Hui B, Shi YH, Zhou J, Peng YF, Gu CY, Yang H, Shi GM, Ke AW, Wang XY et al. 2011 Autophagy activation in hepatocellular carcinoma contributes to the tolerance of oxaliplatin via reactive oxygen species modulation. Clinical Cancer Research 17 6229-6238. (doi:10.1158/1078-0432.CCR-11-0816)

Feng Z, Zhang H, Levine AJ \& Jin S 2005 The coordinate regulation of the $\mathrm{p} 53$ and mTOR pathways in cells. PNAS 102 8204-8209. (doi:10.1073/pnas.0502857102)

Frankel LB, Wen J, Lees M, Hoyer-Hansen M, Farkas T, Krogh A, Jaattela M \& Lund AH 2011 microRNA-101 is a potent inhibitor of autophagy. EMBO Journal 30 4628-4641. (doi:10.1038/emboj.2011.331)

Gao X, Zacharek A, Salkowski A, Grignon DJ, Sakr W, Porter AT \& Honn KV 1995 Loss of heterozygosity of the BRCA1 and other loci on chromosome 17q in human prostate cancer. Cancer Research 55 1002-1005.

Glick D, Barth S \& Macleod KF 2010 Autophagy: cellular and molecular mechanisms. Journal of Pathology 221 3-12. (doi:10.1002/path.2697)

Grasso CS, Wu Y-M, Robinson DR, Cao X, Dhanasekaran SM, Khan AP, Quist MJ, Jing X, Lonigro RJ, Brenner JC et al. 2012 The mutational landscape of lethal castration-resistant prostate cancer. Nature 487 239-243. (doi:10.1038/ nature11125)

Gundara J, Robinson B \& Sidhu S 2011 Evolution of the "autophagamiR". Autophagy 7 12. (doi:10.4161/auto.7. 12.17762)

Gutierrez MG, Saka HA, Chinen I, Zoppino FCM, Yoshimori T, Bocco JL \& Colombo MI 2007 Protective role of autophagy against Vibrio cholerae cytolysin, a pore-forming toxin from V. cholerae. PNAS 104 1829-1834. (doi:10.1073/pnas. 0601437104)

Hashimoto I, Koizumi K, Tatematsu M, Minami T, Cho S, Takeno N, Nakashima A, Sakurai H, Saito S, Tsukada K et al. 2008 Blocking on the CXCR4/mTOR signalling pathway induces the anti-metastatic properties and autophagic cell death in peritoneal disseminated gastric cancer cells. European Journal of Cancer 44 1022-1029. (doi:10.1016/j.ejca.2008.02.043)

Huang Y, Guerrero-Preston R \& Ratovitski E 2012 PhosphoDeltaNp63alpha-dependent regulation of autophagic signaling through transcription and micro-RNA modulation. Cell Cycle 11 1247-1259. (doi:10.4161/cc.11.6. 19670)
Jung HS \& Lee M-S 2010 Role of autophagy in diabetes and mitochondria. Annals of the New York Academy of Sciences 1201 79-83. (doi:10.1111/j.1749-6632.2010. 05614.x)

Karantza-Wadsworth V, Patel S, Kravchuk O, Chen G, Mathew R, Jin S \& White E 2007 Autophagy mitigates metabolic stress and genome damage in mammary tumorigenesis. Genes and Development 21 1621-1635. (doi:10.1101/gad.1565707)

Kim MS, Song SY, Lee JY, Yoo NJ \& Lee SH 2011 Expressional and mutational analyses of ATG5 gene in prostate cancers. APMIS 119 802-807.

Kishimoto H, Hamada K, Saunders M, Backman S, Sasaki T, Nakano T, Mak TW \& Suzuki A 2003 Physiological functions of Pten in mouse tissues. Cell Structure and Function 28 11-21. (doi:10.1247/csf.28.11)

Klionsky DJ \& Emr SD 2000 Autophagy as a regulated pathway of cellular degradation. Science 290 1717-1721. (doi:10.1126/science.290.5497.1717)

Klionsky DJ, Abeliovich H, Agostinis P, Agrawal DK, Aliev G, Askew DS, Baba M, Baehrecke EH, Bahr BA, Ballabio A et al. 2008 Guidelines for the use and interpretation of assays for monitoring autophagy in higher eukaryotes. Autophagy 4 151-175.

Koneri K, Goi T, Hirono Y, Katayama K \& Yamaguchi A 2007 Beclin 1 gene inhibits tumor growth in colon cancer cell lines. Anticancer Research 27 1453-1457.

Korkmaz G, le Sage C, Tekirdag KA, Agami R \& Gozuacik D 2012 miR-376b controls starvation and mTOR inhibitionrelated autophagy by targeting ATG4C and BECN1. Autophagy 8 165-176. (doi:10.4161/auto.8.2.18351)

Kovaleva V, Mora R, Park YJ, Plass C, Chiramel AI, Bartenschlager R, Dohner H, Stilgenbauer S, Pscherer A, Lichter P et al. 2012 miRNA-130a targets ATG2B and DICER1 to inhibit autophagy and trigger killing of chronic lymphocytic leukemia cells. Cancer Research 72 1763-1772. (doi:10.1158/0008-5472.CAN-11-3671)

Kroemer G, Galluzzi L, Vandenabeele P, Abrams J, Alnemri ES, Baehrecke EH, Blagosklonny MV, El-Deiry WS, Golstein P, Green DR et al. 2009 Classification of cell death: recommendations of the Nomenclature Committee on Cell Death 2009. Cell Death and Differentiation 16 3-11. (doi:10.1038/cdd.2008.150)

Kuma A, Hatano M, Matsui M, Yamamoto A, Nakaya H, Yoshimori T, Ohsumi Y, Tokuhisa T \& Mizushima N 2004 The role of autophagy during the early neonatal starvation period. Nature 432 1032-1036. (doi:10.1038/ nature03029)

Le X-F, Mao W, Lu Z, Carter BZ \& Bast RC Jr 2010 Dasatinib induces autophagic cell death in human ovarian cancer. Cancer 116 4980-4990. (doi:10.1002/cncr.25426)

Li B-X, Li C-Y, Peng R-Q, Wu X-J, Wang H-Y, Wan D-S, Zhu X-F \& Zhang X-S 2009 The expression of beclin 1 is associated with favorable prognosis in stage IIIB colon cancers. Autophagy 5 303-306. (doi:10.4161/auto.5.3.7491) 
Li J, Hou N, Faried A, Tsutsumi S \& Kuwano H $2010 a$ Inhibition of autophagy augments 5-fluorouracil chemotherapy in human colon cancer in vitro and in vivo model. European Journal of Cancer 46 1900-1909. (doi:10.1016/j.ejca.2010.02.021)

Li J, Wang X-L, Fang Y-C \& Wang C-Y 2010 $b$ Tephrosininduced autophagic cell death in A549 non-small cell lung cancer cells. Journal of Asian Natural Products Research 12 992-1000. (doi:10.1080/10286020.2010.513034)

Li Y, Zhang J, Chen X, Liu T, He W, Chen Y \& Zeng X 2012 Molecular machinery of autophagy and its implication in cancer. American Journal of the Medical Sciences $\mathbf{3 4 3}$ 155-161. (doi:10.1097/MAJ.0b013e31821f978d)

Liang XH, Jackson S, Seaman M, Brown K, Kempkes B, Hibshoosh H \& Levine B 1999 Induction of autophagy and inhibition of tumorigenesis by beclin 1 . Nature $\mathbf{4 0 2}$ 672-676. (doi:10.1038/45257)

Lin C-I, Whang EE, Abramson MA, Jiang X, Price BD, Donner DB, Moore FD Jr \& Ruan DT 2009 Autophagy: a new target for advanced papillary thyroid cancer therapy. Surgery 146 1208-1214. (doi:10.1016/j.surg.2009.09.019)

Liu J-J, Lin M, Yu J-Y, Liu B \& Bao J-K 2011 Targeting apoptotic and autophagic pathways for cancer therapeutics. Cancer Letters 300 105-114. (doi:10.1016/j. canlet.2010.10.001)

Lomonaco SL, Finniss S, Xiang C, Decarvalho A, Umansky F, Kalkanis SN, Mikkelsen T \& Brodie C 2009 The induction of autophagy by gamma-radiation contributes to the radioresistance of glioma stem cells. International Journal of Cancer 125 717-722. (doi:10.1002/ijc.24402)

Lu Z, Luo RZ, Lu Y, Zhang X, Yu Q, Khare S, Kondo S, Kondo Y, Yu Y, Mills GB et al. 2008 The tumor suppressor gene ARHI regulates autophagy and tumor dormancy in human ovarian cancer cells. Journal of Clinical Investigation 118 3917-3929.

Miracco C, Cosci E, Oliveri G, Luzi P, Pacenti L, Monciatti I, Mannucci S, De Nisi MC, Toscano M, Malagnino V et al. 2007 Protein and mRNA expression of autophagy gene Beclin 1 in human brain tumours. International Journal of Oncology 30 429-436.

Mujumdar N, Mackenzie TN, Dudeja V, Chugh R, Antonoff MB, Borja-Cacho D, Sangwan V, Dawra R, Vickers SM \& Saluja AK 2010 Triptolide induces cell death in pancreatic cancer cells by apoptotic and autophagic pathways. Gastroenterology 139 598-608. (doi:10.1053/j.gastro.2010.04.046)

Mukubou H, Tsujimura T, Sasaki R \& Ku Y 2010 The role of autophagy in the treatment of pancreatic cancer with gemcitabine and ionizing radiation. International Journal of Oncology 37 821-828.

Pezzolesi MG, Platzer P, Waite KA \& Eng C 2008 Differential expression of PTEN-targeting microRNAs miR-19a and miR-21 in Cowden syndrome. American Journal of Human Genetics 82 1141-1149. (doi:10.1016/ j.ajhg.2008.04.005)
Powell MJ, Casimiro MC, Cordon-Cardo C, He X, Yeow W-S, Wang C, McCue PA, McBurney MW \& Pestell RG 2011 Disruption of a Sirt1-dependent autophagy checkpoint in the prostate results in prostatic intraepithelial neoplasia lesion formation. Cancer Research 71 964-975.

Qu X, Yu J, Bhagat G, Furuya N, Hibshoosh H, Troxel A, Rosen J, Eskelinen E-L, Mizushima N, Ohsumi Y et al. 2003 Promotion of tumorigenesis by heterozygous disruption of the beclin 1 autophagy gene. Journal of Clinical Investigation 112 1809-1820.

Ravikumar B, Sarkar S, Davies JE, Futter M, GarciaArencibia M, Green-Thompson ZW, Jimenez-Sanchez M, Korolchuk VI, Lichtenberg M, Luo S et al. 2010 Regulation of mammalian autophagy in physiology and pathophysiology. Physiological Reviews 90 1383-1435. (doi:10.1152/physrev.00030.2009)

Roccaro AM, Sacco A, Jia X, Azab AK, Maiso P, Ngo HT, Azab F, Runnels J, Quang P \& Ghobrial IM 2010 microRNA-dependent modulation of histone acetylation in Waldenstrom macroglobulinemia. Blood 116 1506-1514. (doi:10.1182/blood-2010-01-265686)

Russell SE, Hickey GI, Lowry WS, White P \& Atkinson RJ 1990 Allele loss from chromosome 17 in ovarian cancer. Oncogene 5 1581-1583.

Saito H, Inazawa J, Saito S, Kasumi F, Koi S, Sagae S, Kudo R, Saito J, Noda K \& Nakamura Y 1993 Detailed deletion mapping of chromosome 17q in ovarian and breast cancers: 2-cM region on 17q21.3 often and commonly deleted in tumors. Cancer Research 53 3382-3385.

Shen Y, Li D-D, Wang L-L, Deng R \& Zhu X-F 2008 Decreased expression of autophagy-related proteins in malignant epithelial ovarian cancer. Autophagy 4 1067-1068.

Shen S, Kepp O, Michaud M, Martins I, Minoux H, Metivier D, Maiuri MC, Kroemer RT \& Kroemer G 2011 Association and dissociation of autophagy, apoptosis and necrosis by systematic chemical study. Oncogene 30 4544-4556. (doi:10.1038/onc.2011.168)

Shen S, Kepp O \& Kroemer G 2012 The end of autophagic cell death? Autophagy 8 1-3. (doi:10.4161/auto.8.1. 16618)

Shenouda SK \& Alahari SK 2009 MicroRNA function in cancer: oncogene or a tumor suppressor? Cancer Metastasis Reviews 28 369-378. (doi:10.1007/s10555009-9188-5)

Takeuchi H, Kondo Y, Fujiwara K, Kanzawa T, Aoki H, Mills GB \& Kondo S 2005 Synergistic augmentation of rapamycin-induced autophagy in malignant glioma cells by phosphatidylinositol 3-kinase/protein kinase B inhibitors. Cancer Research 65 3336-3346.

Ullen A, Farnebo M, Thyrell L, Mahmoudi S, Kharaziha P, Lennartsson L, Grander D, Panaretakis T \& Nilsson S 2010 Sorafenib induces apoptosis and autophagy in prostate cancer cells in vitro. International Journal of Oncology 37 15-20. (doi:10.3892/ijo_00000648) 
Vanderlaag K, Su Y, Frankel AE, Burghardt RC, Barhoumi R, Chadalapaka G, Jutooru I \& Safe S 2010 1,1-Bis ( $3^{\prime}$-indolyl)-1-(p-substituted phenyl)methanes induce autophagic cell death in estrogen receptor negative breast cancer. BMC Cancer 10669 . (doi:10.1186/ 1471-2407-10-669)

Vazquez-Martin A, Oliveras-Ferraros C \& Menendez JA 2009 Autophagy facilitates the development of breast cancer resistance to the anti-HER2 monoclonal antibody trastuzumab. PLOS ONE 4 e6251. (doi:10.1371/journal. pone.0006251)

Wang Z-H, Xu L, Duan Z-L, Zeng L-Q, Yan N-H \& Peng Z-L 2007 Beclin 1-mediated macroautophagy involves regulation of caspase-9 expression in cervical cancer HeLa cells. Gynecologic Oncology 107 107-113. (doi:10.1016/j.ygyno.2007.05.034)

Wang N, Feng Y, Zhu M, Tsang C-M, Man K, Tong Y \& Tsao S-W 2010 Berberine induces autophagic cell death and mitochondrial apoptosis in liver cancer cells: the cellular mechanism. Journal of Cellular Biochemistry 111 1426-1436. (doi:10.1002/jcb.22869)

White E \& DiPaola R 2009 The double-edged sword of autophagy modulation in cancer. Clinical Cancer Research 15 5308-5316. (doi:10.1158/1078-0432.CCR07-5023)

White E, Karp C, Strohecker AM, Guo Y \& Mathew R 2010 Role of autophagy in suppression of inflammation and cancer. Current Opinion in Cell Biology 22 212-217. (doi:10.1016/j.ceb.2009.12.008)

Xu S \& Weihua Z 2011 Loss of EGFR induced autophagy sensitizes hormone refractory prostate cancer cells to adriamycin. Prostate 71 1216-1224. (doi:10.1002/pros. 21337)
Yang S \& Kimmelman AC 2011 A critical role for autophagy in pancreatic cancer. Autophagy 7 912-913. (doi:10.4161/ auto.7.8.15762)

Yang Z \& Klionsky DJ 2010 Mammalian autophagy: core molecular machinery and signaling regulation. Current Opinion in Cell Biology 22 124-131. (doi:10.1016/j.ceb. 2009.11.014)

Yang Q \& Mao Z 2010 Parkinson disease: a role for autophagy? Neuroscientist 16 335-341. (doi:10.1177/ 1073858409357118)

Yu Y, Yang L, Zhao M, Zhu S, Kang R, Vernon P, Tang D \& Cao L 2012 Targeting microRNA-30a-mediated autophagy enhances imatinib activity against human chronic myeloid leukemia cells. Leukemia 26 1752-1760 (doi: 10.1038/leu.2012.65). (doi:10.1038/leu.2012.65)

Zhai H, Song B, Xu X, Zhu W \& Ju J 2012 Inhibition of autophagy and tumor growth in colon cancer by miR-502. Oncogene 14 167. (doi:10.1111/j.1744-4667.2012.00113.x)

Zhu H, Wu H, Liu X, Li B, Chen Y, Ren X, Liu C-G \& Yang J-M 2009 Regulation of autophagy by a beclin 1-targeted microRNA, miR-30a, in cancer cells. Autophagy $\mathbf{5}$ 816-823.

Zou Z, Wu L, Ding H, Wang Y, Zhang Y, Chen X, Chen X, Zhang C-Y, Zhang Q \& Zen K 2012 MicroRNA-30a sensitizes tumor cells to cis-platinum via suppressing beclin 1-mediated autophagy. Journal of Biological Chemistry $\mathbf{2 8 7}$ 4148-4156. (doi:10.1074/jbc.M111.307405)

Received in final form 1 October 2012 Accepted 16 October 2012 Made available online as an Accepted Preprint 18 October 2012 